\title{
Compound Odontoma
}

\author{
Brenda L. Nelson $\cdot$ Lester D. R. Thompson
}

Received: 20 May 2010/Accepted: 31 May 2010/Published online: 9 June 2010

(C) US Government 2010

\begin{abstract}
A case of compound odontomas of the anterior maxilla is discussed. Multiple tooth like structures were discovered upon surgical excision.
\end{abstract}

Keywords Odontoma $\cdot$ Compound $\cdot$ Maxilla .

Tooth-like

\section{History}

An 8-year-old girl presented to her dentist for a routine dental examination. Radiographs revealed a lesion of the anterior maxilla. Neither the patient nor her parents could recall any history of pain or swelling in the area.

\section{Radiographic Features}

A panographic radiograph showed a collection of toothlike structures with a narrow radiolucent rim apical to the deciduous canine, lateral and central incisors in the right maxilla. The structures have disrupted eruption of both permanent incisors and the right permanent lateral incisor (Fig. 1).

\section{Diagnosis}

The gross specimen consisted of six malformed tooth-like structures. The tissue was decalcified, and processed via

B. L. Nelson $(\varangle) \cdot$ L. D. R. Thompson Department of Anatomic Pathology, Naval Medical Center San Diego, 34800 Bob Wilson Drive, San Diego, CA 92134-5000, USA

e-mail: brenda.nelson@med.navy.mil formalin fixation. Hematoxylin and eosin stained material showed tooth-like structures composed of dentin and cementum with centrally located loose fibrous tissue, representing pulp tissue (Fig. 2). Scant enamel matrix was identified (Fig. 3).

\section{Discussion}

Odontomas are the most common odontogenic tumor. They are considered to be hamartomas rather than neoplasms, and are composed of the tissues native to teeth: enamel, dentin, cementum and pulp tissue. Odontomas are further sub-classified based upon their gross and radiographic features into compound (small tooth like structures) or complex (a conglomeration of dentin, enamel and cementum) [1].

Odontomas may be found at any age; however, most are detected in the first two decades of life. There is no gender predilection and most lesions are detected on routine radiographs. Clinical symptoms are uncommon, however, an affected patient may present when a permanent tooth or multiple teeth that fail to erupt [2]. Rarely, an odontoma may erupt into the oral cavity [3]. Compound odontomas are more commonly found in the anterior maxilla, while complex odontomas tend to favor the posterior mandible. Odontomas are generally small; however, they may occasionally grow large resulting in bone expansion [1].

Usually, odontomas can be confidently subclassified based on the radiographic appearance alone. Compound odontomas appear as a collection of small teeth leaving few entities in the radiographic differential diagnosis except perhaps, a supernumerary tooth. Complex odontomas appear as a radiodense mass of hard tissues which may result in a broader differential diagnosis [3]. Both have 


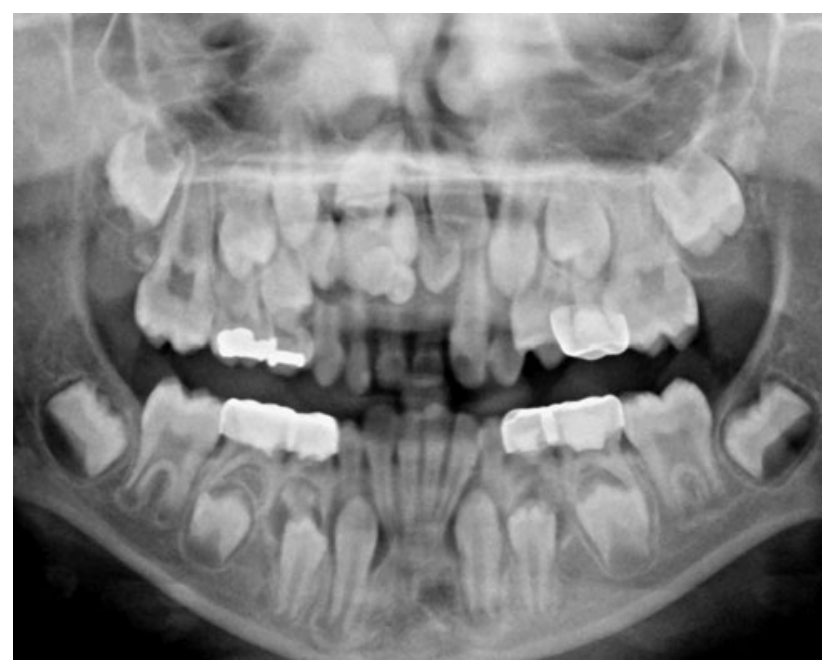

Fig. 1 Panographic radiograph shows a small collection of tooth-like structures in the anterior maxilla

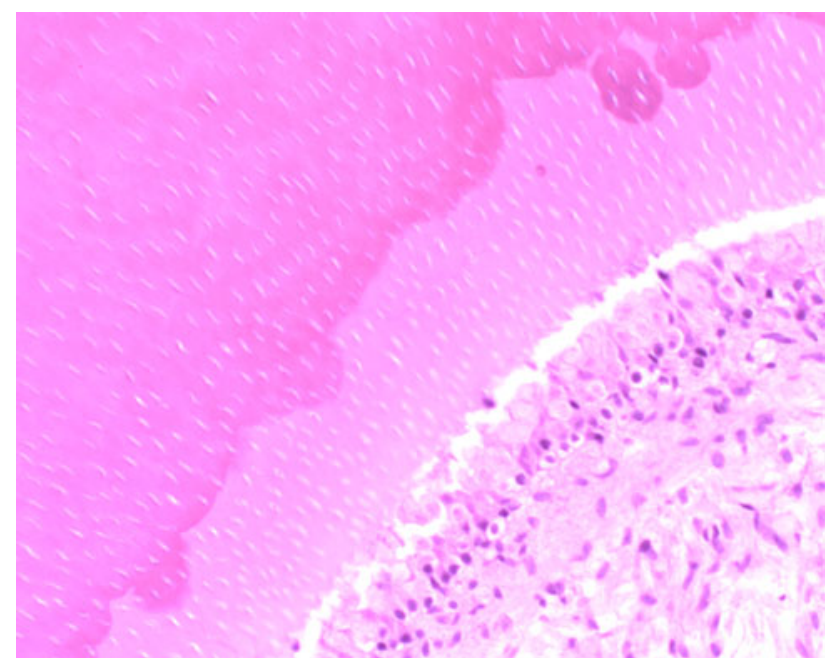

Fig. 2 Photomicograph shows pulpal tissue adjacent to predentin and mature dentin. The relationship is similar to that of normal teeth

radiolucent rims, representing dental follicular tissue or, less commonly, a dentigerous cyst.

Histologically the odontoma is not a diagnostic dilemma. It is composed of dentin, cementum, pulpal tissue and enamel. However, mature enamel is lost during the decalcification processing and will not be seen on conventional hematoxylin and eosin stained slides. The compound odontoma recapitulates the organization of a normal tooth, while the complex odontoma appears as a disorganized

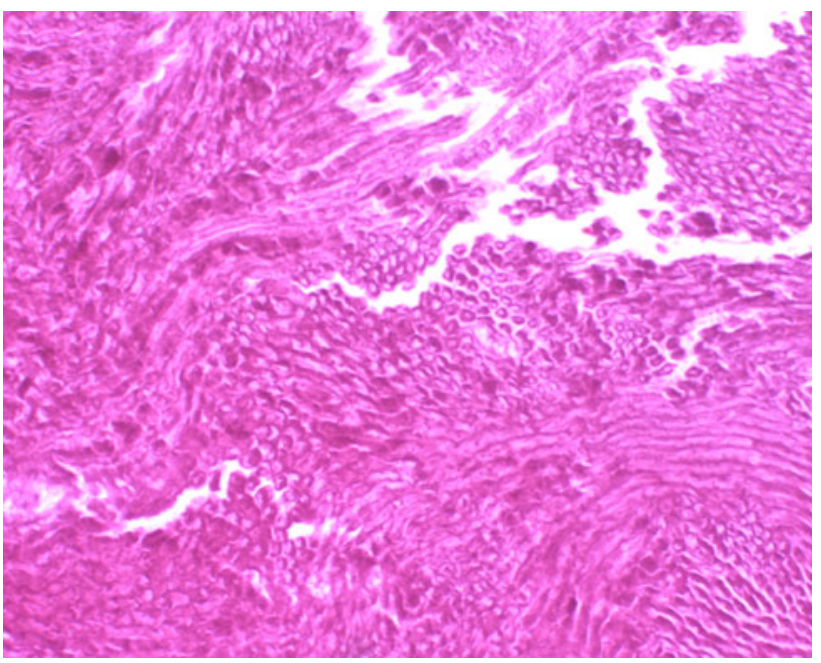

Fig. 3 This image shows enamel matrix with its characteristic "fish scale" appearance. Mature enamel is completely lost to processing

mass of hard odontogenic tissues [1]. Loose, myxoid connective tissue with odontogenic epithelial rests may be seen in close association with the lesion, and most often represents normal dental follicular tissue. Fibrous connective tissue with a cystic lining representing a dentigerous cyst may also be seen.

Treatment for an odontoma is complete removal with any associated soft tissues. Recurrences do not occur. Orthodontic treatment may be indicated to correct any malocclusion.

Disclaimer The opinions and assertions expressed herein are those of the author and are not to be construed as official or representing the views of the Department of the Navy or the Department of Defense.

\section{References}

1. Bordini J Jr, Contar CM, Sarot JR, Fernandes A, Machado MA. Multiple compound odontomas in the jaw: case report and analysis of the literature. J Oral Maxillofac Surg. 2008;66(12):2617-20.

2. Nagaraj K, Upadhyay M, Yadav S. Impacted maxillary central incisor, canine, and second molar with 2 supernumerary teeth and an odontoma. Am J Orthod Dentofacial Orthop. 2009;135(3): $390-9$.

3. Serra-Serra G, Berini-Aytés L, Gay-Escoda C. Erupted odontomas: a report of three cases and review of the literature. Med Oral Patol Oral Cir Bucal. 2009;14(6):E299-303. (Review). 